\title{
Skyrme-Extended-Thomas-Fermi Approach Method In Investigation of Nuclear Ground State Properties of ${ }^{208} \mathrm{~Pb}$
}

\author{
Yacobus Yulianto ${ }^{1, \mathrm{a}}$ and Zaki Su'ud ${ }^{1, \mathrm{~b}}$ \\ ${ }^{1}$ Nuclear and Biophysics Research Division, Physics Department, \\ Faculty of Mathematics and Natural Science, Institut Teknologi Bandung, \\ J1. Ganesha 10 Bandung, 40132, Indonesia. \\ Email: 'ayacyulianto@gmail.com, ${ }^{\mathrm{b}}$ zakisuud@gmail.com
}

Received: 17 March 2016, Revised: 1 June 2016, Accepted: 12 july 2016

\begin{abstract}
In this research, it is performed the nuclear ground state properties investigation of ${ }^{208} \mathrm{~Pb}$ by using the SETF method with SLy4 set parameters. The energy optimization calculation is performed using SETFA code. The SETFA results are in good agreement with the related experiment results, and also with the results of the HFBRAD and HFODDHFBTHO codes. It is can be indicated that Skyrme-Extended-Thomas-Fermi method can be used to explain the nuclear ground state properties, especially even-stable nucleus.
\end{abstract}

Keywords: Extended-Thomas-Fermi, nuclear binding energy, optimization, Skyrme interaction, SLy4

\section{INTRODUCTION}

Studying the nuclear ground state properties microscopically, especially with regard to the nuclear structure, in addition to using Skyrme-Hartree-Fock (SHF), the semiclassical approach has been also shown to be quite accurate, especially the so-called SkyrmeExtended-Thomas-Fermi (SETF) method. This approach method can also be used to describe the nuclear ground state properties [1]. Calculations using SETF approach method can be used to determine the nuclear structure through minimizing total energy by varying the densities of proton and neutron in the framework of selfconsistent [2]. This calculation just requires the integration of the nuclear total energy density. In SETF approach method, the kinetic energy and spin-orbit densities are expressed as a density functional of proton and neutron [3]. Integral calculation results by minimizing energy density will provide information about proton and neutron densities of the nuclear, so that the nuclear ground state properties can be determined, such as the local density, the kinetic energy density, and the spin-orbit density. In this research, it is used SETF method because this method is quite good in explaining the nuclear ground state properties. In addition, the used formula is semi-classical and it uses statistical approaches. However, the result of the calculation is quite able to explain the experiment results and the results obtained by SHF method. It has been demonstrated by previous researchers $[4,5]$.

In this research, ${ }^{208} \mathrm{~Pb}$ was chosen to be studied because the ${ }^{208} \mathrm{~Pb}$ has an even number of nucleons (even nucleus), including the number of proton and neutron. By using even nucleus,

\footnotetext{
Corresponding author.

E-mail address: yacyulianto@gmail.com
} 
the complexity of energy density formula due to the asymmetric effect in relation to the peculiarity number of proton and neutron can be reduced.

The purposes of this research are to calculate numerically the ground state energy of ${ }^{208} \mathrm{~Pb}$ by using the SETF method with SLy4 set parameters and to simulate the local density and the local potential distributions for both proton and neutron of ${ }^{208} \mathrm{~Pb}$.

\section{THEORY}

\section{Skyrme Interaction}

In studying nuclear structure, the used Hamiltonian formula can be expressed in the form:

$\hat{H}=\sum_{i=1}^{A}-\frac{2}{2 m} \nabla_{i}^{2}+\frac{1}{2} \sum_{i, j=1}^{A} \hat{v}_{i j}^{(2) ~ N u c l}+\frac{1}{6} \sum_{i, j, k=1}^{A} \hat{v}_{i j k}^{(3) N u c l}+\frac{1}{2} \sum_{i, j=1}^{Z} \hat{v}_{i j}^{(2) \text { Coul }}$

where the first term is the kinetic part for all nucleons, the second and the third terms are the nucleons interaction, and the fourth term is the Coulomb interaction between protons [6]. The kinetic term (with the addition of center-mass energy correction term) can be expressed as:

$$
\varepsilon_{\text {Kinetik }}=\frac{2}{2 m}\left(1-\frac{1}{A}\right) t
$$

where $t$ is the total kinetic energy density (sum of the proton and neutron kinetic energy densities) and the factor in parentheses describes the one-body part of the center-of-mass correction [7]. The second and the third terms can be approximated by using the Skyrme interaction. This interaction was proposed by T.H.R. Skyrme in 1959, where in the Skyrme model, it is assumed that the potential between the nucleons in nuclear can be represented by the interaction of two or more particles that can be formulated as:

$$
\hat{v}_{C S}=\sum_{i<j} \hat{v}_{i j}^{(2)}+\sum_{i<j<k} \hat{v}_{i j k}^{(3)}
$$

where $\hat{v}_{i j}^{(2)}$ describes the potential of two-body interaction and $\hat{v}_{i j k}^{(3)}$ describes the potential of three-body interaction $[6,8,9]$. The functional energy density can be expressed [9]:

$$
\begin{aligned}
\varepsilon_{\text {Skyme }}= & \frac{1}{2} t_{0}\left[\left(1+\frac{1}{2} x_{0}\right) \rho^{2}-\left(x_{0}+\frac{1}{2}\right)\left(\rho_{n}^{2}+\rho_{p}^{2}\right)\right]+\frac{1}{12} t_{3}\left(1+\frac{1}{2} x_{3}\right) \rho^{2+\alpha} \\
& -\frac{1}{12} t_{3}\left(x_{3}+\frac{1}{2}\right) \rho^{\alpha}\left(\rho_{n}^{2}+\rho_{p}^{2}\right)+\frac{1}{4}\left[t_{1}\left(1+\frac{x_{1}}{2}\right)+t_{2}\left(1+\frac{x_{2}}{2}\right)\right] \rho t \\
& -\frac{1}{4}\left[t_{1}\left(x_{1}+\frac{1}{2}\right)-t_{2}\left(x_{2}+\frac{1}{2}\right)\right]\left(\rho_{n} t_{n}+\rho_{p} t_{p}\right) \\
& +\frac{1}{16}\left[3 t_{1}\left(1+\frac{x_{1}}{2}\right)-t_{2}\left(1+\frac{x_{2}}{2}\right)\right](\nabla \rho)^{2} \\
& -\frac{1}{16}\left[3 t_{1}\left(x_{1}+\frac{1}{2}\right)+t_{2}\left(x_{2}+\frac{1}{2}\right)\right]\left[\left(\nabla \rho_{p}\right)^{2}+\left(\nabla \rho_{n}\right)^{2}\right] \\
& -\frac{1}{16}\left[t_{1} x_{1}+t_{2} x_{2}\right] J^{2}+\frac{1}{16}\left(t_{1}-t_{2}\right)\left(J_{p}^{2}+J_{n}^{2}\right) \\
& -\frac{1}{2} W_{o}\left(\rho \nabla \cdot J+\rho_{n} \nabla \cdot J_{n}+\rho_{p} \nabla \cdot J_{p}\right)
\end{aligned}
$$

where is the reduced Planck constant, $m$ is the mass of the nucleon, $t_{p(n)}$ is the kinetic energy density of proton (p) or neutron (n), and $J_{p(n)}$ is the spin-orbit density for proton or neutron [9]. Coulomb term is expressed in equation [11]:

$$
\varepsilon_{\text {Coul }}=\frac{e^{2}}{2} \int \frac{\rho_{p}(r) \rho_{p}\left(r^{\prime}\right)}{\left|r-r^{\prime}\right|} d^{3} r^{\prime}-\frac{3}{4} e^{2}\left(\frac{3}{\pi}\right)^{1 / 3} \rho_{p}^{4 / 3}(r)
$$

Based on the formulation of nucleon interaction in the Skyrme model above, the nuclear total energy density is a function of the local density $\rho_{q}(r)$, the kinetic energy density $t_{q}(r)$, and the spin-orbit density $J_{p}(r)$. The total energy, integrated from the total energy density over the space, can be formulated as:

$E=\int\left(\varepsilon_{\text {Kinetik }}+\varepsilon_{\text {Skyrme }}+\varepsilon_{\text {Coul }}\right) d^{3} r=\int\left[\varepsilon_{\text {Kinetik }}(\rho, t, J)\right] d^{3} r$

(6)

From the formulation of the energy density in eq. (4), it can be derived the formula for the central potential of particles as [2]:

$$
\begin{aligned}
V_{q}(r)= & t_{0}\left[\left(1+\frac{x_{0}}{2}\right) \rho-\left(x_{0}+\frac{1}{2}\right) \rho_{q}\right]+\frac{1}{4}\left[t_{1}\left(1+\frac{x_{1}}{2}\right)+t_{2}\left(1+\frac{x_{2}}{2}\right)\right] t \\
& -\frac{1}{4}\left[t_{1}\left(x_{1}+\frac{1}{2}\right)-t_{2}\left(x_{2}+\frac{1}{2}\right)\right] t_{q}-\frac{1}{8}\left[3 t_{1}\left(1+\frac{x_{1}}{2}\right)-t_{2}\left(1+\frac{x_{2}}{2}\right)\right]\left(\nabla^{2} \rho\right) \\
& +\frac{1}{8}\left[3 t_{1}\left(x_{1}+\frac{1}{2}\right)+t_{2}\left(x_{2}+\frac{1}{2}\right)\right]\left(\nabla^{2} \rho_{q}\right)+(2+\alpha) \rho^{\alpha+1} \frac{t_{3}}{12}\left(1+\frac{x_{3}}{2}\right) \\
& -\frac{1}{12} t_{3}\left(\frac{1}{2}+x_{3}\right)\left[\alpha \rho^{\alpha-1}\left(\rho_{p}^{2}+\rho_{n}^{2}\right)+2 \rho^{\alpha} \rho_{q}\right] \\
& -\frac{1}{2} W_{0}\left[\nabla \cdot J+\nabla \cdot J_{q}\right]+V_{\text {Coul }}(r) \delta_{p q}
\end{aligned}
$$


where [12]

$$
V_{\text {Coul }}(r)=e^{2} \int d^{3} r \frac{\rho_{p}\left(r^{\prime}\right)}{\left|r-r^{\prime}\right|}-e^{2}\left(\frac{1}{\pi}\right)^{1 / 3} \rho_{p}^{1 / 3}(r)
$$

Tabel 1. The SLy4 set parameters for Skyrme interaction

\begin{tabular}{cccc}
\hline Parameter & SLy4 & Parameter & SLy4 \\
\hline$t_{0}$ & -2488 & $x_{0}$ & 0.834 \\
$t_{1}$ & 486.82 & $x_{1}$ & -0.344 \\
$t_{2}$ & -546.3 & $x_{2}$ & -1 \\
$t_{3}$ & 13777 & $x_{3}$ & 1.354 \\
$W_{0}$ & 123 & $\alpha$ & 0.167 \\
\hline
\end{tabular}

The parameters of $t_{0}, t_{1}, t_{2}, t_{3}, x_{0}, x_{1}, x_{2}, x_{3}, \alpha$, dan $W_{0}$ in eq. (4) and (7) are the parameters for the Skyrme interaction which are usually determined such that the ground state properties of stable nuclei obtained experimentally can be explained. In this research, it is utilized the SLy4 set parameters because SLy4 is suitable to explain the ground state properties of neutronrich nuclei.

\section{EXTENDED THOMAS FERMI APPROACH METHODS}

It can be seen from eq. (6) that the total energy density depends on the local density $\rho_{q}(r)$, the kinetic energy density $t_{q}(r)$, and the spin-orbit density $J_{p}(r)$, where $q$ represents proton or neutron. Solutions with purely quantum mechanical approach are quite complex. Therefore, it is needed an alternative method which is more simple and accurate enough to solve nuclear many-body problem. The method (which is used in this study) is socalled Skyrme-Extended-Thomas-Fermi (SETF) method. In SETF method, the density of kinetic energy which can be expressed:

$t_{q}^{(E T F)}\left[\rho_{q}\right]=t_{q}^{(T F)}\left[\rho_{q}\right]+t_{q}^{(2)}\left[\rho_{q}\right]+t_{q}^{(4)}\left[\rho_{q}\right]+t_{q}^{(4) S O}\left[\rho_{q}\right]$

(9)

The formulation of the spin-orbit density is expressed as:

$$
J_{q}\left[\rho_{q}\right]=J_{q}^{[2]}\left(\rho_{q}\right)+J_{q}^{[4]}\left(\rho_{q}\right)
$$

The complete formula of eq. (9) and eq. (10) can be found in following references $[2,5,10]$. After the entire quantities of kinetic energy density and spin-orbit density are expressed as a function of the local density, it can be calculated the eq. (6) by applying the variational methods using the nucleon local density as a varied quantity, which follows [2]:

$$
\delta E=\delta \int \varepsilon\left[\rho_{n}(r), \rho_{p}(r)\right] d^{3} r=0
$$

Nucleon density of the nuclear in ground state can be approximated by adopting the FermiDirac distribution formula and it can be formulated as:

$$
\rho_{q}(r)=\rho_{0_{q}}\left[1+\exp \left(\frac{r-R_{0 q}}{a_{q}}\right)\right]^{-\gamma_{q}}, \quad q=\{n, p\}
$$

The nucleon density parameter $\rho_{0 q}$ can be determined by the terms of normalized

$$
\int \rho_{q}(r) d^{3} r=Z(N)
$$

The rms (root-mean-square) radii for proton and neutron are defined as [7]:

$$
r_{q}=\left\langle r_{q}^{2}\right\rangle^{\frac{1}{2}}=\left[\frac{\int r^{2} \rho_{q}(r) d^{3} r}{\int \rho_{q}(r) d^{3} r}\right]^{\frac{1}{2}}
$$

\section{CALCULATION METHOD}

In this research, optimizing of eq. (12) is performed in order to obtain the optimum total energy value of ${ }^{208} \mathrm{~Pb}$. It is utilized the finitedifference method for numerical differential calculation and the Riemann sum for numerical integration calculation. To optimize the value of the total energy density, it is used Nelder-Mead method. To help the calculation process, it is developed a code which is named SETFA code. In this code, it is implemented the complete formula for kinetic energy density and spin orbit density. For kinetic energy density, as described by eq. (9), there are additional terms, i.e. correction for fourth order and correction for fourth order because of spin-orbit. For spin-orbit density, it is used second and fourth order correction, as described by eq. (10). To verify the calculation results of this simple code, it is used the calculation results from HFBRAD code [7], HFODD-HFBTHO code [14], and experiment [15]. 


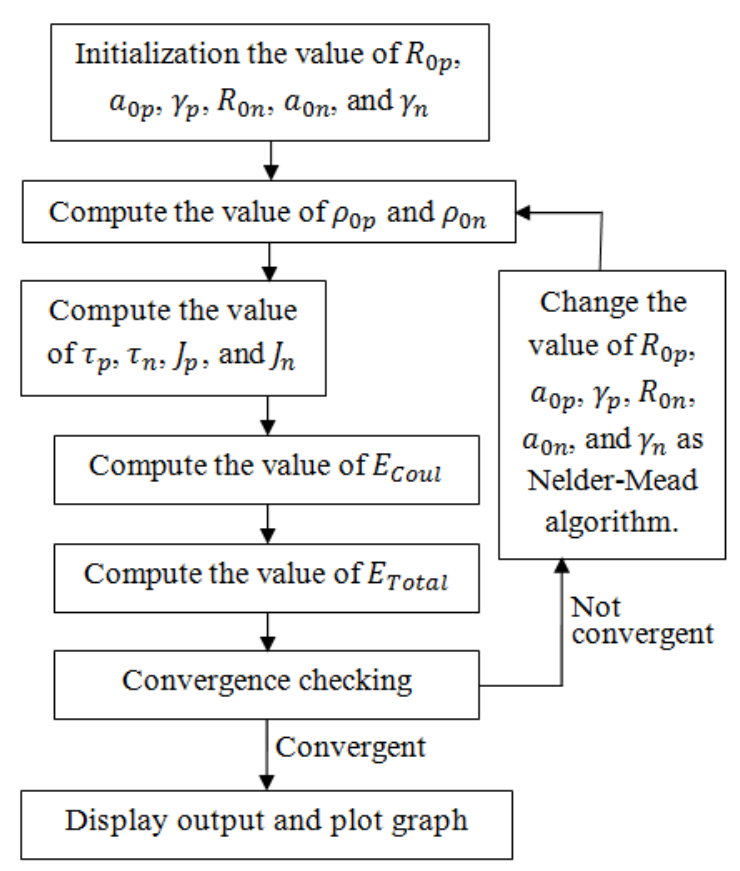

Fig. 1. Flowchart of SEFTA code

\section{RESULT AND DISCUSSION \\ Calculation Results}

From the optimization calculation, the obtained values of the parameters that describe the ground state properties of ${ }^{208} \mathrm{~Pb}$ are shown in Table 2. From Table 3, it is shown the calculation results of rms radius of proton and neutron in ${ }^{208} \mathrm{~Pb}$ using Skyrme interaction with SLy4 set parameters for SETFA, HAFOMN, and HFODD-HFBTHO results.

From Table 4, it is shown that there are significant differences in total energy value, where the HFBRAD and HFODD-HFBTHO program results are quite close to the energy experiments. It can be also seen that spin-orbit energy of SETFA result is lowest with discrepancy about $16 \mathrm{MeV}$ compared with both HFBRAD and HFODD-HFBTHO results because the HFBRAD and the HFODDHFBTHO codes have added the pairing energy and the rearrangement energy terms, whereas in this study, those energies have not inserted yet because it is still on-going research. From these results, it is seen that the energy value obtained by using SETF method is quite close to experiment energy with $0.882363 \%$ of discrepancy.

Table 2. The Calculation Results

\begin{tabular}{|c|c|}
\hline Parameter & Nilai \\
\hline $\begin{array}{l}\text { Proton density distribution } \\
\text { radius } R_{0 p}(\mathrm{fm})\end{array}$ & 6.97705 \\
\hline $\begin{array}{l}\text { Neutron density distribution } \\
\text { radius } R_{0 n}(\mathrm{fm})\end{array}$ & 7.18385 \\
\hline $\begin{array}{l}\text { Proton density diffusivity } \\
a_{0 p}(\mathrm{fm})\end{array}$ & 0.52826 \\
\hline $\begin{array}{l}\text { Neutron density diffusivity } \\
a_{0 n}(\mathrm{fm})\end{array}$ & 0.65034 \\
\hline Proton density exponent $\gamma_{p}$ & 1.4361 \\
\hline Neutron density exponent $\gamma_{n}$ & 1.5291 \\
\hline $\begin{array}{l}\text { Proton density parameter } \\
\rho_{0 p}\left(\mathrm{fm}^{-3}\right)\end{array}$ & 0.06247816 \\
\hline $\begin{array}{l}\text { Neutron density parameter } \\
\rho_{0 n}\left(\mathrm{fm}^{-3}\right)\end{array}$ & 0.09076248 \\
\hline Kinetic Energy (MeV) & 3870.722380 \\
\hline Skyrme Energy (MeV) & -6289.721860 \\
\hline Spin-Orbit Energy (MeV) & -80.528340 \\
\hline $\begin{array}{l}\text { Direct Coulomb Energy } \\
(\mathrm{MeV})\end{array}$ & 827.876513 \\
\hline $\begin{array}{l}\text { Coulomb Exchange Energy } \\
(\mathrm{MeV})\end{array}$ & -31.393103 \\
\hline $\begin{array}{l}\text { Coulomb Total Energy } \\
(\mathrm{MeV})\end{array}$ & 796.483410 \\
\hline Total Energy (MeV) & -1622.516070 \\
\hline Experiment Energy (MeV) & $-1636,96$ \\
\hline Discrepancy (\%) & 0.882363 \\
\hline
\end{tabular}

Table 3. The calculation results of rms radius of proton and neutron in ${ }^{208} \mathrm{~Pb}$ using Skyrme interaction with SLy4 set parameters.

\begin{tabular}{lccc}
\hline Parameter & S-ETF & HFBRAD & $\begin{array}{c}\text { HFODD- } \\
\text { HFBTHO }\end{array}$ \\
\hline $\begin{array}{l}\text { Proton rms } \\
\text { radius (fm) }\end{array}$ & 5.452905 & 5.45765 & 5.4580 \\
$\begin{array}{l}\text { Neutron } \\
\text { rms radius } \\
(\mathrm{fm})\end{array}$ & 5.628432 & 5.61745 & 5.6180 \\
\hline
\end{tabular}


Table 4. The comparison of energy calculation results of ${ }^{208} \mathrm{~Pb}$.

\begin{tabular}{|c|c|c|}
\hline Energy & Program & Nilai (MeV) \\
\hline \multirow[t]{3}{*}{ Kinetic } & $\begin{array}{l}\text { HFODD- } \\
\text { HBTHO }\end{array}$ & 3865.317805 \\
\hline & HFBRAD & 3866.171870 \\
\hline & SETFA & 3870.722380 \\
\hline \multirow[t]{3}{*}{ Spin-Orbit } & $\begin{array}{l}\text { HFODD- } \\
\text { HBTHO }\end{array}$ & -96.418201 \\
\hline & HFBRAD & -96.443690 \\
\hline & SETFA & -80.528341 \\
\hline \multirow[t]{3}{*}{ Direct Coulomb } & $\begin{array}{l}\text { HFODD- } \\
\text { HBTHO }\end{array}$ & 827.840920 \\
\hline & HFBRAD & 827.903090 \\
\hline & SETFA & 827.876513 \\
\hline \multirow[t]{3}{*}{ Exchange Coulomb } & $\begin{array}{l}\text { HFODD- } \\
\text { HBTHO }\end{array}$ & -31.265702 \\
\hline & HFBRAD & -31.268400 \\
\hline & SETFA & -31.393103 \\
\hline \multirow[t]{3}{*}{ Total } & $\begin{array}{l}\text { HFODD- } \\
\text { HBTHO }\end{array}$ & -1635.531496 \\
\hline & HFBRAD & -1635.673178 \\
\hline & SETFA & -1622.516070 \\
\hline Experiment [15] & & -1636.96 \\
\hline
\end{tabular}

\section{Distribution Models}

It is shown by Fig. 2 that the local density distribution for the proton in ${ }^{208} \mathrm{~Pb}$ obtained by using SETFA code is in good agreement with local proton density distribution obtained by using the SHFB method, especially for the slope and surface regions. In other hand, the value of the local density of proton and neutron at 0-4 fm is different because each method has different theoretical approach to model the nuclear structure.
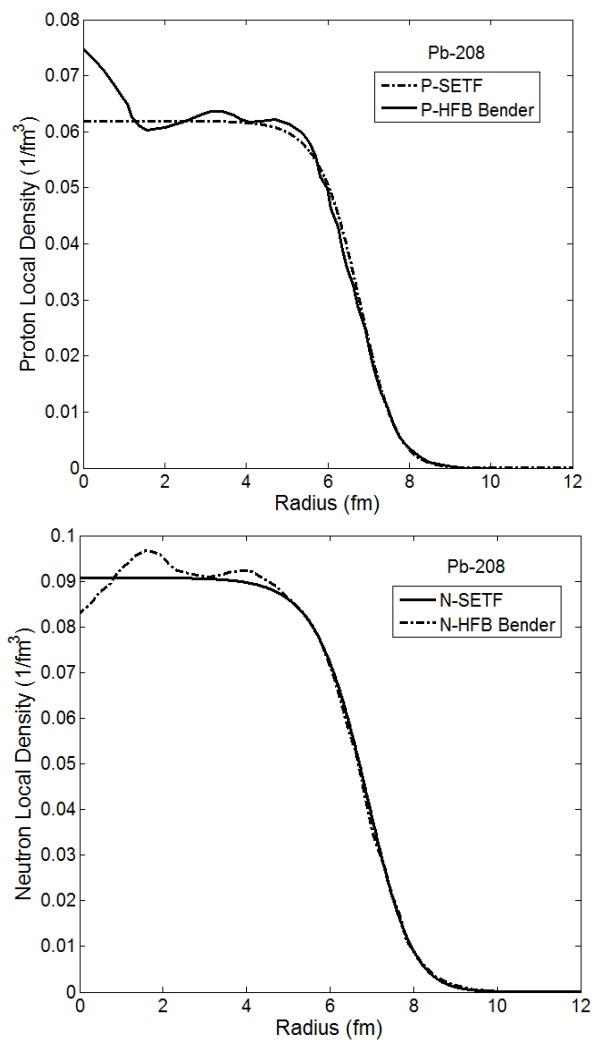

Fig. 2. The distribution models of the local density of proton (top) and neutron (bottom) of ${ }^{208} \mathrm{~Pb}$.
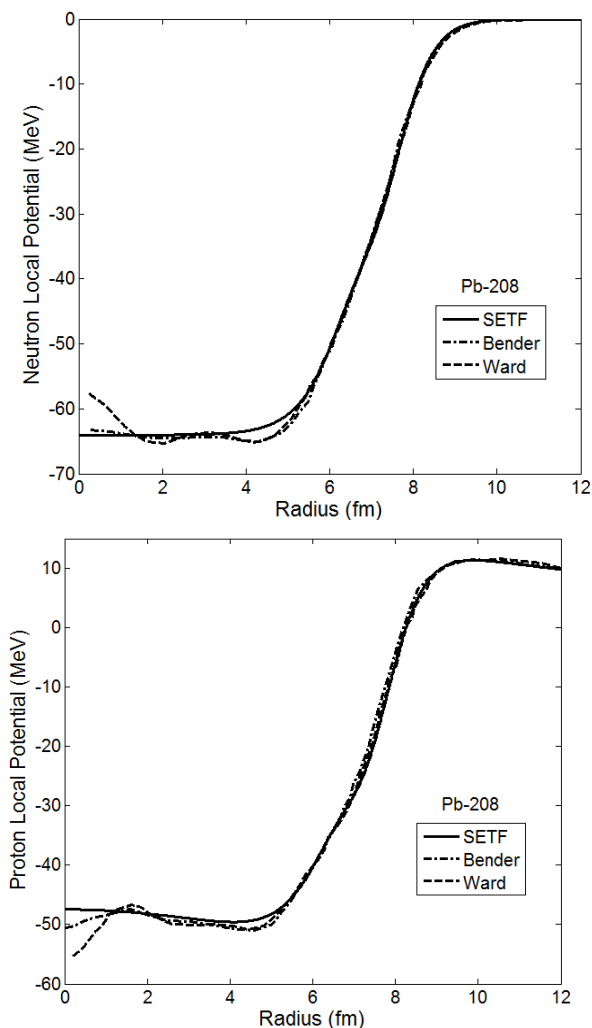

Fig. 3. The distribution models of the local potential of proton (left) and neutron (right) of

${ }^{208} \mathrm{~Pb}$. The solid line represents this research calculation result, the dotted-dash line represents the calculation result of Bender et al. [16], and the dotted line represents the calculation result of Ward et al. [17]. 
For the local potential distribution, it is also compared our results with the results of Bender et al. and Ward et al. From Fig. 3, it is seen that the potential depth of both proton and neutron for all three results are very similar to each other, although each method used different set parameters. In this research, it is used SETF method with SLy4 set parameters, Bender et al. used SHF method with SLy6 set parameters, and Ward et al. used SHFB method with SLy6 set parameters.

From those figures above, it can be seen that there are differences between SETF and SHFB results at near the center. It is because in SETF method, it is used statistical approach to determine local density of nucleons, and in SHFB method, it is used the nucleon's wave function to determine local density of nucleons. Since the obtained results of SETF and SHFB methods are similar, especially the slope and the surface regions, it can be indicated that SETF method can be used to study the nuclear ground state properties, especially for even-stable nuclei.

\section{SUMMARY}

In this research, it has been calculated the total energy calculations of ${ }^{208} \mathrm{~Pb}$ by using Skyrme-Extended-Thomas-Fermi

(SETF) approach method with SLy4 set parameters. A SETFA code has been also developed to perform calculations process. The obtained results of this research have been compared with the calculation results of the related experiment and the other researchers, where the results of this research are in good agreement with the results of related experiment and the other researchers. It can be indicated that Skyrme-ExtendedThomas-Fermi method can be used to study the ground state properties of nuclear, especially for even-stable nucleus.

\section{REFERENCES}

[1] M. Brack, C. Guet, and H. Hakanson, Selfconsistent semiclassical description of average nuclear properties - A link between microscopic and macroscopic models, Phys. Rep. 123 (5), 275, 1985.

[2] J. Bartel and K. Bencheikh, Nuclear mean fields through self-consistent semi classical calculations, Eur. Phys. J. A 14, 179, 2002.

[3] V. Denisov and V. Nesterov, Distribution of density and potential of nuclear interaction, Ukr. J. Phys. 51 (5), 440, 2006.

[4] A. Baran, K. Pomorski, and J. Bartel, Extended Thomas-Fermi estimates of single particle potentials for doubly magic and super heavy nuclei, Ann. Univ. Marie Curie 57 (2), 23, 2002.

[5] A. Dobrowolski, K. Pomorski, and J. Bartel, Mean-field description of fusion barriers with Skyrme's interaction, Nucl. Phys. A 729, 713, 2003.

[6] P. Ring and P. Shuck, The nuclear many body problem, Springer, Berlin, 149, 1980.

[7] K. Bennaceur and J. Dobaczewski, Coordinate-space solution of the SkyrmeHartree-Fock-Bogolyubov equations within spherical symmetry. The program HFBRAD (v1.00), Comp. Phys. Communications 168, 96, 2005.

[8] T.H.R. Skyrme, The effective nuclear potential, Nucl. Phys. 9, 615, 1959.

[9] D. Vautherin and D.M. Brink, HartreeFock Calculation with Skyrme's interaction I. Spherical nuclei, Phys. Rev. C 5, 626, 1972.

[10] E. Chabanat, et al., A Skyrme parametrization from subnuclear to neutron star densities, Nucl. Phys. A 627, 710, 1997.

[11] H.Q. Gu, et al., Slater approximation for Coulomb exchange effects in nuclear covariant density functional theory, Phys. Rev. C 87, 041301, 2013.

[12] F. Aymard, F. Gulminelli, and J. Margueron, In-medium nuclear cluster energies within the Extended ThomasFermi approach, Phys. Rev. C 89, 065807, 2014.

[13] V. Denisov and V. Nesterov, Distribution of nucleus-nucleus potential and difuseness of density distribution in nuclei, Ukr. J. Phys. UDC 539.17, 108, 2006.

[14] N. Schunck, et al., Solution of the SkyrmeHartree-Fock-Bogolyubov equations in the cartesian deformed harmonic-oscillator basis. (VII) HFODD (v2.49t): A new 
version of the Program, Comp. Phys. Communications 183, 166, 2012.

[15] M. Wang et al., The AME2012 atomic mass evolution (II). Tables, graphs and references, Chinese Physics. C 36, 1603, 2012.

[16] M. Bender, P.H. Heenen, and P.G. Reinhard, Self-consistent mean-field models for nuclear structure, Reviews of Modern Physics 75, 121, 2003.

[17] D.E. Ward, B.G. Carlsson, and S. Aberg, $\alpha$-decay calculations of heavy nuclei using an effective Skyrme interaction, Phys. Rev. $C$ 88, 064316, 2013. 